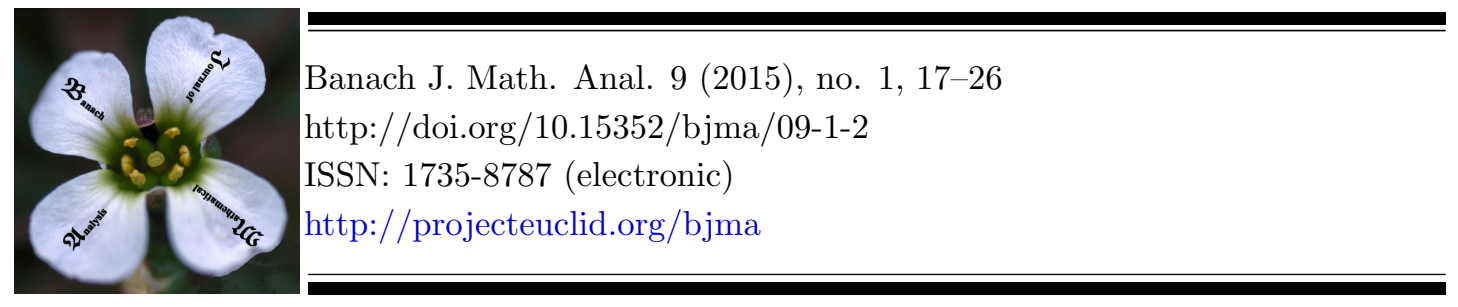

\title{
SOME RESULTS ON MATRIX POLYNOMIALS IN THE MAX ALGEBRA
}

\author{
NEDA GHASEMIZADEH ${ }^{1}$ AND GHOLAMREZA AGHAMOLLAEI ${ }^{2 *}$ \\ Communicated by P. Y. Wu
}

\begin{abstract}
For any $n \times n$ nonnegative matrix $A$, and any norm $\|\cdot\|$ on $\mathbb{R}^{n}$, $\eta_{\|\cdot\|}(A)$ is defined as $\sup \left\{\frac{\|A \otimes x\|}{\|x\|}: x \in \mathbb{R}_{+}^{n}, x \neq 0\right\}$. Let $P(\lambda)$ be a matrix polynomial in the max algebra. In this paper, we introduce $\eta_{\|.\|}[P(\lambda)]$, as a generalization of the matrix norm $\eta_{\|.\|}($.$) , and we investigate some algebraic$ properties of this notion. We also study some properties of the maximum circuit geometric mean of the companion matrix of $P(\lambda)$ and the relationship between this concept and the matrices $P(1)$ and coefficients of $P(\lambda)$. Some properties of $\eta_{\|.\|}(\Psi)$, for a bounded set of max matrix polynomials $\Psi$, are also investigated.
\end{abstract}

\section{INTRODUCTION AND PRELIMINARIES}

The max algebra consists of the set (semiring) of nonnegative real numbers equipped with the basic operations of multiplication $a \otimes b=a b$, and maximization $a \oplus b=\max \{a, b\}$. In fact, the algebraic system max algebra and its isomorphic versions provide an attractive way of describing a class of nonlinear problems appearing for instance in manufacturing and transportation scheduling, information technology, discrete event-dynamic systems, combinatorial optimization, mathematical physics and etc., see, e.g., [3], [6], [7], [8], [11] and [13] and references cited there, for a description of such systems and their applications. During the

Date: Received: Nov. 19, 2013; Accepted: Jan. 14, 2014.

* Corresponding author.

2010 Mathematics Subject Classification. Primary 15A80; Secondary 47A30, 47A56, 15A60, $15 \mathrm{~A} 18$.

Key words and phrases. Matrix polynomials, Max algebra, Nonnegative matrices, maximum circuit geometric mean. 
last years, the max version of the classical concepts about matrices has attention, and several results have been obtained; see e.g., [4], [5], [9], [10], [15], [17] and [19].

Let $\mathbb{R}_{+}$be the set of all nonnegative real numbers and $M_{m \times n}\left(\mathbb{R}_{+}\right)$be the set of all $m \times n$ nonnegative real matrices. The notions $M_{n}\left(\mathbb{R}_{+}\right)$and $\mathbb{R}_{+}^{n}$ are considered for $M_{n \times n}\left(\mathbb{R}_{+}\right)$and $M_{n \times 1}\left(\mathbb{R}_{+}\right)$, respectively. $M_{m \times n}(\mathbb{R})$, by analogy with $M_{n \times n}\left(\mathbb{R}_{+}\right)$, is considered. For $A=\left(a_{i j}\right) \in M_{n}(\mathbb{R})$, we say $A$ is positive (nonnegative) and write $A>0(A \geq 0)$ if $a_{i j}>0\left(a_{i j} \geq 0\right)$ for $1 \leq i, j \leq n$. Let $A=\left(a_{i j}\right) \in M_{m \times n}\left(\mathbb{R}_{+}\right)$ and $B=\left(b_{i j}\right) \in M_{n \times k}\left(\mathbb{R}_{+}\right)$. The product of $A$ and $B$ in the max algebra is denoted by $A \otimes B$, and $(A \otimes B)_{i j}=\max _{k=1, \ldots, n}\left(a_{i k} b_{k j}\right)$. The notation $A_{\otimes}^{2}$ means $A \otimes A$, and $A_{\otimes}^{k}$ denotes the $k$ th power of $A$. If $B=\left(b_{i j}\right) \in M_{m \times n}\left(\mathbb{R}_{+}\right)$, then the sum of $A$ and $B$ in the max algebra is denoted by $A \oplus B$ and $(A \oplus B)_{i j}=\max \left\{a_{i j}, b_{i j}\right\}$. For $A \in M_{n}\left(\mathbb{R}_{+}\right)$, the weighted directed graph $D(A)$ associated with $A$ has vertices $1, \ldots, n$, and there is an edge from $i$ to $j$ with weight $a_{i j}$ if and only if $a_{i j}>0$. A path of length $k$ is a sequence of $k$ edges $\left(i_{1}, i_{2}\right),\left(i_{2}, i_{3}\right), \ldots,\left(i_{k}, i_{k+1}\right)$. A circuit of length $k$ is a sequence of $k$ edges $\left(i_{1}, i_{2}\right),\left(i_{2}, i_{3}\right), \ldots,\left(i_{k-1}, i_{k}\right),\left(i_{k}, i_{1}\right)$, where $i_{1}, \ldots, i_{k}$ are distinct. This is a path with $i_{k+1}=i_{1}$ and $i_{1}, \ldots, i_{k}$ are distinct. Associated with this circuit is the circuit geometric mean known as $\left(a_{i_{1} i_{2}} a_{i_{2} i_{3}} \ldots a_{i_{k} i_{1}}\right)^{\frac{1}{k}}$. Note that circuits $\left(i_{1}, i_{1}\right)$ of length 1 (loops) are included here and that we also consider empty circuits, i.e., circuits that consist of only one vertex and have length zero. For empty circuits, the associated circuit geometric mean is zero. The maximum circuit geometric mean in $D(A)$ is denoted by $\mu(A)$. A circuit with circuit geometric mean equal to $\mu(A)$ is called a critical circuit. For more details see [14, Chapter 8].

Let $A \in M_{n}\left(\mathbb{R}_{+}\right)$. A scalar $\lambda$ is called a right max eigenvalue of $A$ if $A \otimes x=\lambda x$ for some nonnegative vector $x \neq 0$, namely, $\max _{1 \leq j \leq n}\left(a_{i j} x_{j}\right)=\lambda x_{i} ; i=1,2, \ldots, n$. The vector $x$ is called a right max eigenvector of $A$ corresponding to $\lambda$. In this sense, $(\lambda, x)$ is called a right max eigenpair of $A$. The set of all right max eigenvalues of $A$ is called the max spectrum of $A$, and denoted by $\sigma_{m}(A)$. Also a scalar $\lambda$ is called a left max eigenvalue of $A$ if $x^{T} \otimes A=\lambda x^{T}$ for some nonnegative vector $x \neq 0$. The vector $x$ is called a left max eigenvector of $A$ corresponding to $\lambda$. In this sense, $(\lambda, x)$ is called a left max eigenpair of $A$. The following theorem, which is known as the max version of the Perron-Ferbenius theorem(see [14, Theorem 8.4.4]), is important in the context of matrices over the max algebra.

Theorem 1.1. [4]; Let $A \in M_{n}\left(\mathbb{R}_{+}\right)$be an irreducible matrix. Then $\mu(A)$ is the unique max eigenvalue of $A$ and every corresponding max eigenvector is positive.

At the end of this section, we study matrix polynomials in the max algebra; for more information see [12]. Suppose that

$$
P(\lambda)=A_{0} \oplus \lambda A_{1} \oplus \cdots \oplus \lambda^{m-1} A_{m-1}
$$

is a max matrix polynomial, where $A_{0}, A_{1}, \ldots, A_{m-1}$ are in $M_{n}\left(\mathbb{R}_{+}\right), \lambda \in \mathbb{R}_{+}$, and $m$ is a positive integer. The numbers $(m-1)$ and $n$ are considered as the degree and the order of $P(\lambda)$, respectively. Let $k, \tau \in \mathbb{R}_{+}$. We say, see e.g., [12], that 
(1) The real number $k \geq 0$ is a right max eigenvalue of $P(\lambda)$ with corresponding right max eigenvector $v \geq 0$ if $P(k) \otimes v=k^{m} v$. In this sense, $(k, v)$ is called a right max eigenpair of $P(\lambda)$.

(2) The real number $\tau \geq 0$ is a left max eigenvalue of $P(\lambda)$ with corresponding left max eigenvector $w \geq 0$ if $w^{T} \otimes P(\tau)=\tau^{m} w^{T}$. In this sense, $(\tau, w)$ is called a left max eigenpair of $P(\lambda)$.

The set of all right max eigenvalues of the max matrix polynomial $P(\lambda)$ as in (1.1), is called the max spectrum of $P(\lambda)$, and is denoted by $\sigma_{m}[P(\lambda)]$.

The companion matrix of $P(\lambda)$ is defined as

$$
C_{P}=\left(\begin{array}{ccccc}
0 & I & \cdots & 0 & 0 \\
0 & 0 & \cdots & 0 & 0 \\
\vdots & \vdots & \ddots & \vdots & \vdots \\
0 & 0 & \cdots & 0 & I \\
A_{0} & A_{1} & \cdots & A_{m-2} & A_{m-1}
\end{array}\right) \in M_{n m}\left(\mathbb{R}_{+}\right) .
$$

Proposition 1.2. [12]; Let $P(\lambda)$ be a max matrix polynomial as in (1.1), and $C_{P}$ as in (1.2), be the companion matrix of $P(\lambda)$. Then $(k, v) \in \mathbb{R}_{+} \times \mathbb{R}_{+}^{n}$ is a right max eigenpair of $P(\lambda)$ if and only if $(k, \hat{v}) \in \mathbb{R}_{+} \times \mathbb{R}_{+}^{m n}$ is a right max eigenpair of $C_{P}$, where

$$
\hat{v}=\left(\begin{array}{c}
v \\
k v \\
\vdots \\
k^{m-1} v
\end{array}\right)
$$

Also, $(\tau, w) \in \mathbb{R}_{+} \times \mathbb{R}_{+}^{n}$ is a left max eigenpair of $P(\lambda)$ if and only if $(\tau, \hat{w}) \in$ $\mathbb{R}_{+} \times \mathbb{R}_{+}^{m n}$ is a left max eigenpair of $C_{P}$, where

$$
\hat{w}=\left(\begin{array}{c}
\frac{1}{\tau} A_{0}^{T} \otimes w \\
\left(\frac{1}{\tau^{2}} A_{0}^{T} \oplus \frac{1}{\tau} A_{1}^{T}\right) \otimes w \\
\vdots \\
\left(\frac{1}{\tau^{m-1}} A_{0}^{T} \oplus \frac{1}{\tau^{m-2}} A_{1}^{T} \oplus \cdots \oplus \frac{1}{\tau} A_{m-2}^{T}\right) \otimes w \\
w
\end{array}\right) .
$$

Consequently, $\sigma_{m}[P(\lambda)]=\sigma_{m}\left(C_{P}\right)$.

The study of matrix polynomials in the conventional algebra has along history, and they arise in many applications and their spectral analysis is very important when studying linear systems of ordinary differential equations with constant coefficients, and also stability theory; see e.g., [1, 2] and [18] and references cited there. In this paper, we study some results about matrix polynomials in the max algebra. The emphasis is on the relationship between the maximum circuit geometric mean of the max matrix polynomial $P(\lambda)$ as in $(1.1)$, namely $\mu\left(C_{P}\right)$, where $C_{P}$ is as in (1.2), and the matrices $P(1)$, and $A_{0}, A_{1}, \ldots, A_{m-1}$. Some properties of $\eta_{\|\cdot\|}(\Psi)$, for a bounded set of max matrix polynomials $\Psi$, are also investigated. 


\section{MAIN RESUlTS}

Associated with any norm $\|$.$\| on \mathbb{R}^{n}$ and $A \in M_{n}\left(\mathbb{R}_{+}\right)$, the notion of $\eta_{\|\cdot\|}(A)$ was first introduced by Y.Y. Lur in [15] (see also [16]) as

$$
\eta_{\|\cdot\|}(A)=\sup \left\{\frac{\|A \otimes x\|}{\|x\|}: x \in \mathbb{R}_{+}^{n}, x \neq 0\right\} .
$$

It is the max version of the definition of operator norm in functional analysis. He used this notation to study the max algebra version of the joint spectral radius, and some other concepts. Now, we introduce this notion for matrix polynomials in the max algebra.

Definition 2.1. Let $P(\lambda)=A_{0} \oplus \lambda A_{1} \oplus \cdots \oplus \lambda^{m-1} A_{m-1}$ be a max matrix polynomial as in (1.1), and $\|$.$\| be a vector norm on \mathbb{R}^{n}$. The symbol $\eta_{\|.\|}[P(\lambda)]$ is defined as

$$
\eta_{\|\cdot\|}[P(\lambda)]:=\max \left\{\eta_{\|\cdot\|}\left(A_{0}\right), \ldots, \eta_{\|\cdot\|}\left(A_{m-1}\right)\right\}
$$

where $\eta_{\|.\|}($.$) is as in (2.1).$

Next we want to state some properties of $\eta_{\|\cdot\|}($.$) for max matrix polynomials.$ We recall that a norm $\|\cdot\|$ on $\mathbb{R}^{n}$ is called monotone if the inequality $|x| \leq|y|$ implies that $\|x\| \leq\|y\|$ for all $x, y \in \mathbb{R}^{n}$. Also, the induced matrix norm by $\|$. on $M_{n}(\mathbb{R})$ is defined by $\|A\|=\max _{\|x\|=1}\|A x\|$, where $A \in M_{n}(\mathbb{R})$, see e.g., [14, p. 292]. If $A_{0}, \ldots, A_{m-1}$ are matrices in $M_{n}\left(\mathbb{R}_{+}\right)$, the multigraph associated with $A_{0}, \ldots, A_{m-1}$ is denoted by $M$. Thus, $M$ consists of the vertices $\{1, \ldots, n\}$ with an edge of weight $a_{i j}^{(p)}$ from $i$ to $j$ for every $p$, where $a_{i j}^{(p)}>0$ is $(i, j)$-entry of $A_{p}$ for $p=0, \ldots, m-1$. A cycle in the multigraph $M$ is a sequence of vertices $i_{1}, i_{2}, \ldots, i_{k}, i_{k+1}=i_{1}$, where $i_{1}, i_{2}, \ldots, i_{k}$ are distinct and edges with weights $a_{i_{j} i_{j+1}}^{\left(p_{j}\right)}>0$, where $j=1,2, \ldots, k$, and $p_{1}, p_{2}, \ldots, p_{k}$ are in $\{0, \ldots, m-1\}$. The geometric mean of a cycle in $M$ and the maximal cycle geometric mean $\mu(M)$ of $M$ are defined as similar to a simple graph; see section 1 , and for more information see [12].

In the following propositions, we study the relationship between $\mu\left(C_{P}\right)$ and $\mu\left(A_{j}\right)$, where $P(\lambda)=A_{0} \oplus \lambda A_{1} \oplus \cdots \oplus \lambda^{m-1} A_{m-1}$ is a max matrix polynomial as in (1.1) with the companion matrix $C_{P}$ as in (1.2).

Proposition 2.2. Let $P(\lambda)=A_{0} \oplus \lambda A_{1} \oplus \cdots \oplus \lambda^{m-1} A_{m-1}$ be a max matrix polynomial as in (1.1). Then $\max \left\{\mu\left(A_{0}\right), \ldots, \mu\left(A_{m-1}\right)\right\} \leq \mu(P(1))$.

Proof. Let $M$ be the multigraph associated with nonnegative matrices

$$
A_{0}, \ldots, A_{m-1},
$$

and $\mu(M)$ denote the maximal cycle geometric mean of $M$. It is clear that $\max \left\{\mu\left(A_{0}\right), \ldots, \mu\left(A_{m-1}\right)\right\} \leq \mu(M)$. So the result follows from [12, Lemma 5.1].

Proposition 2.3. Let $P(\lambda)=A_{0} \oplus \lambda A_{1} \oplus \cdots \oplus \lambda^{m-1} A_{m-1}$ be a max matrix polynomial as in (1.1) with the companion matrix $C_{P}$ as in (1.2). If $\mu(P(1)) \leq 1$, then $\max \left\{\mu\left(A_{0}\right), \ldots, \mu\left(A_{m-1}\right)\right\} \leq \mu\left(C_{P}\right)$. 
Proof. By [12, Corollary 5.2], $\mu(P(1)) \leq \mu\left(C_{P}\right)$. So, the result follows from Proposition 2.2.

Proposition 2.4. Let $P(\lambda)=A_{0} \oplus \lambda A_{1} \oplus \cdots \oplus \lambda^{m-1} A_{m-1}$ be a max matrix polynomial as in (1.1) with the companion matrix $C_{P}$ as in (1.2). If $\mu\left(C_{P}\right)=$ $\mu(P(1))<1$, then

$$
\mu\left(C_{P}\right)=\max \left\{\mu\left(A_{0}\right), \ldots, \mu\left(A_{m-1}\right)\right\}=\mu\left(A_{m-1}\right) .
$$

Proof. Proposition 2.3 implies that $\max \left\{\mu\left(A_{0}\right), \ldots, \mu\left(A_{m-1}\right)\right\} \leq \mu\left(C_{P}\right)$. Since $\mu\left(C_{P}\right)=\mu(P(1))$ and $\mu(P(1)) \neq 1$, by [12, Corollary 5.3], $\mu\left(C_{P}\right)=\mu\left(A_{m-1}\right)$. Therefore,

$$
\max \left\{\mu\left(A_{0}\right), \ldots, \mu\left(A_{m-1}\right)\right\} \leq \mu\left(C_{P}\right)=\mu\left(A_{m-1}\right) \leq \max \left\{\mu\left(A_{0}\right), \ldots, \mu\left(A_{m-1}\right)\right\} .
$$

Hence, the result holds.

Next to study $\eta_{\|.\|[}[$.$] for max matrix polynomials, we need the following Lemma.$

Lemma 2.5. Let $A, B \in M_{n}\left(\mathbb{R}_{+}\right)$, and $\|$.$\| be a monotone norm on \mathbb{R}^{n}$. Then

$$
\eta_{\|\cdot\|}(A \oplus B) \leq \eta_{\|\cdot\|}(A)+\eta_{\|\cdot\|}(B) .
$$

Proof. We know that $a \oplus b=\frac{a+b}{2}+\frac{|a-b|}{2}$ for any $a, b \in \mathbb{R}$. Let $x, y \in \mathbb{R}_{+}^{n}$ be arbitrary. Since $\|\cdot\|$ is monotone and $|x-y| \leq x+y,\||x-y|\| \leq\|x+y\|$, and hence,

$$
\|x \oplus y\| \leq\left\|\frac{x+y}{2}\right\|+\left\|\frac{|x-y|}{2}\right\| \leq \frac{\|x\|+\|y\|}{2}+\frac{\|x\|+\|y\|}{2}=\|x\|+\|y\| .
$$

So, by (2.1) and the fact:

$$
(A \oplus B) \otimes x=(A \otimes x) \oplus(B \otimes x)
$$

the result holds.

The sum and product of two max matrix polynomials:

$$
P(\lambda)=A_{0} \oplus \lambda A_{1} \oplus \cdots \oplus \lambda^{m-1} A_{m-1} \text { and } Q(\lambda)=B_{0} \oplus \lambda B_{1} \oplus \cdots \oplus \lambda^{m-1} B_{m-1},
$$

are defined and denoted, respectively, by

$$
P(\lambda) \oplus Q(\lambda)=C_{0} \oplus \lambda C_{1} \oplus \cdots \oplus \lambda^{m-1} C_{m-1} \text {, where } C_{j}=A_{j} \oplus B_{j}
$$

$$
P(\lambda) \otimes Q(\lambda)=C_{0} \oplus \lambda C_{1} \oplus \cdots \oplus \lambda^{2 m-2} C_{2 m-2} \text {, where } C_{j}=\oplus_{i=0}^{j}\left(A_{i} \otimes B_{j-i}\right) .
$$

The notation $(P(\lambda))_{\otimes}^{k}$ means $\underbrace{P(\lambda) \otimes \cdots \otimes P(\lambda)}_{k \text {-times }}$.

Theorem 2.6. Let $P(\lambda)=A_{0} \oplus \lambda A_{1} \oplus \cdots \oplus \lambda^{m-l} A_{m-1}$, and $Q(\lambda)=B_{0} \oplus \lambda B_{1} \oplus$ $\cdots \oplus \lambda^{m-1} B_{m-1}$ be two max matrix polynomials, and $\|$.$\| be a monotone norm on$ $\mathbb{R}^{n}$. Then the following assertions are true:

(1) $\eta_{\|.\|}[P(\lambda)]=0 \Leftrightarrow P(\lambda)=0$;

(2) $\eta_{\|.\|}[\alpha P(\lambda)]=|\alpha| \eta_{\|.\|}[P(\lambda)]$, where $\alpha \in \mathbb{R}$;

(3) If $\mu\left(C_{P}\right)=\mu(P(1))<1$, then $\mu\left(C_{P}\right) \leq \eta_{\|.\|}[P(\lambda)]$; 
(4) $\eta_{\|.\|}[P(\lambda) \oplus Q(\lambda)] \leq \eta_{\|\cdot\|}[P(\lambda)]+\eta_{\|.\|}[Q(\lambda)]$, where $P(\lambda) \oplus Q(\lambda)$ is as in (2.2);

(5) $\eta_{\|.\|}[P(\lambda) \otimes Q(\lambda)] \leq(2 m-1) \eta_{\|.\|}[P(\lambda)] \eta_{\|.\|}[Q(\lambda)]$, where $P(\lambda) \otimes Q(\lambda)$ is as in (2.3).

Proof. Definition 2.1 is used to prove all parts.

By [15, Lemma 1(ii) and (iii)], the results in (1) and (2) are trivial.

To prove (3), by Proposition 2.4 and [15, Lemma $1(\mathrm{vi})]$, we have $\mu\left(C_{P}\right)=$ $\mu\left(A_{m-1}\right) \leq \eta_{\|.\|}\left(A_{m-1}\right)$. So the result holds.

By Lemma 2.5, the proof of (4) is clear.

To prove $(5)$, let $P(\lambda) \otimes Q(\lambda)=C_{0} \oplus \lambda C_{1} \oplus \cdots \oplus \lambda^{2 m-2} C_{2 m-2}$, where $C_{j}=$ $\oplus_{i=0}^{j}\left(A_{i} \otimes B_{j-i}\right)$. Using Lemma 2.5 and [15, Lemma $\left.1(\mathrm{v})\right]$, we have

$$
\begin{aligned}
\eta_{\|\cdot\|}\left(C_{j}\right) & \leq \sum_{i=0}^{j} \eta_{\|\cdot\|}\left(A_{i} \otimes B_{j-i}\right) \\
& \leq \sum_{i=0}^{j} \eta_{\|\cdot\|}\left(A_{i}\right) \eta_{\|\cdot\|}\left(B_{j-i}\right) \\
& \leq(j+1) \eta_{\|\cdot\|}[P(\lambda)] \eta_{\|\cdot\|}[Q(\lambda)] .
\end{aligned}
$$

Hence, the result holds.

Suppose $V=\left\{P(\lambda): P(\lambda)=A_{0} \oplus \lambda A_{1} \oplus \cdots \oplus \lambda^{m} A_{m}\right.$, where $m \in \mathbb{N} \cup\{0\}$, $\left.\lambda \in \mathbb{R}, A_{j} \in M_{n}(\mathbb{R})\right\}$. We define the operation $\oplus$ on $V$ as in (2.2). It is clear that $V$ is a real vector space. Let $\|$.$\| be a norm on \mathbb{R}^{n}$ and $P(\lambda) \in V$. We define

$$
\|P(\lambda)\|=\max \left\{\left\|A_{0}\right\|, \ldots,\left\|A_{m-1}\right\|\right\},
$$

where $\left\|A_{j}\right\|$ is the induced matrix norm by the vector norm $\|$.$\| . Clearly, \|$.$\| is a$ vector norm on the vector space $V$. So, $V$ is a real normed vector space. Next, the following inequality, that is established for max matrix polynomial, is similar to $[15$, Lemma 2].

Proposition 2.7. Let $\|$.$\| be a norm on \mathbb{R}^{n}$. Then there exist positive constants $K_{1}$ and $K_{2}$ such that for every $P(\lambda) \in V$,

$$
K_{1} \eta_{\|\cdot\|}[P(\lambda)] \leq\|P(\lambda)\| \leq K_{2} \eta_{\|\cdot\|}[P(\lambda)] .
$$

Proof. By [15, Lemma 2], there exist $K_{1}>0$ and $K_{2}>0$ such that

$$
K_{1} \eta_{\|\cdot\|}(A) \leq\|A\| \leq K_{2} \eta_{\|\cdot\|}(A)
$$

for any $n \times n$ nonnegative matrix $A$. Let $P(\lambda)=A_{0} \oplus \lambda A_{1} \oplus \cdots \oplus \lambda^{m-1} A_{m-1} \in V$ be given. So,

$$
K_{1} \eta_{\|\cdot\|}\left(A_{i}\right) \leq\left\|A_{i}\right\| \leq K_{2} \eta_{\|\cdot\|}\left(A_{i}\right),
$$

for all $i=1, \ldots, m-1$. Now the result follows from Definition 2.1 and relation $(2.4)$. 
Associated with any norm $\|$.$\| on \mathbb{R}^{n}$ and $A \in M_{n}\left(\mathbb{R}_{+}\right)$, the following notion was first introduced by Y.-Y. Lur in 2005, see e.g, [16], as

$$
\hat{\eta}_{\|.\|}(A)=\limsup _{k \rightarrow \infty}\left[\eta_{\|\cdot\|}\left(A_{\otimes}^{k}\right)\right]^{\frac{1}{k}} .
$$

Now, we introduce this notion for max matrix polynomials.

Definition 2.8. Let $P(\lambda)=A_{0} \oplus \lambda A_{1} \oplus \cdots \oplus \lambda^{m-1} A_{m-1}$ be a max matrix polynomial as in (1.1), and $\|$.$\| be a vector norm on \mathbb{R}^{n}$. We define

$$
\hat{\eta}_{\|\cdot\|}[P(\lambda)]=\max \left\{\hat{\eta}_{\|\cdot\|}\left(A_{0}\right), \ldots, \hat{\eta}_{\|\cdot\|}\left(A_{m-1}\right)\right\},
$$

where $\hat{\eta}_{\|.\|}($.$) is as in (2.5)$.

In the following theorem, we study the relationship between $\mu\left(C_{P}\right)$ with $\eta_{\|.\|}[P(\lambda)]$ and $\hat{\eta}_{\|.\|}[P(\lambda)]$.

Theorem 2.9. Let $P(\lambda)=A_{0} \oplus \lambda A_{1} \oplus \cdots \oplus \lambda^{m-1} A_{m-1}$ be a max matrix polynomial as in (1.1) with the companion matrix $C_{P}$ as in (1.2). If $\mu\left(C_{P}\right)=\mu(P(1))<1$, then the following assertions are true:

(1) $\eta_{\|.\|}[P(\lambda)]<1$ for some norm $\|$.$\| on \mathbb{R}^{n}$;

(2) $\hat{\eta}_{\|.\|}[P(\lambda)]<1$ for some norm $\|$.$\| on \mathbb{R}^{n}$.

Proof. Using Proposition 2.4, we have $\mu\left(A_{i}\right)<1$ for all $i=0,1, \ldots, m-1$. Then by [16, Theorem 2], there exists a norm $\|$.$\| on \mathbb{R}^{n}$ such that $\eta_{\|.\|}\left(A_{i}\right)<1$ and $\hat{\eta}_{\|\cdot\|}\left(A_{i}\right)<1$. Hence, by Definitions 2.1 and 2.8, the results hold.

Proposition 2.10. Let $P(\lambda)=A_{0} \oplus \lambda A_{1} \oplus \cdots \oplus \lambda^{m-1} A_{m-1}$ be a max matrix polynomial as in (1.1) with the companion matrix $C_{P}$ as in (1.2). Then $\eta_{\|.\|}[P(\lambda)]<1$ for some norm $\|$.$\| on \mathbb{R}^{n}$ if and only if $\hat{\eta}_{\|\cdot\|}[P(\lambda)]<1$ for some norm $\|$.$\| on \mathbb{R}^{n}$.

Proof. By [16, Theorem 2] and Definitions 2.1 and 2.8, the result is trivial.

At the end of this section, we want to introduce and study $\eta_{\|\cdot\|}(\Psi)$ for a set $\Psi$ of matrix polynomials in the max algebra. For this mind, let $k$ be a positive integer. The notion $\Psi_{\otimes}^{k}$ denotes the following set:

$$
\Psi_{\otimes}^{k}=\left\{P_{1}(\lambda) \otimes \cdots \otimes P_{k}(\lambda): P_{i}(\lambda) \in \Psi, i=1, \ldots, k\right\} .
$$

Let $\|$.$\| be a norm on \mathbb{R}^{n}$. We know that $\Psi$ is bounded if there exists a $M>0$ such that $\|P(\lambda)\| \leq M$ for all $P(\lambda) \in \Psi$. Hence, in view of Proposition 2.7, $\Psi$ is bounded if and only if there exists a $M>0$ such that $\eta_{\|\cdot\|}[P(\lambda)] \leq M$ for all $P(\lambda) \in \Psi$.

Definition 2.11. Let $\|$.$\| be a norm on \mathbb{R}^{n}$, and $\Psi$ be a bounded set of max matrix polynomials. The notion of $\eta_{\|.\|}(\Psi)$ is defined as :

$$
\eta_{\|\cdot\|}(\Psi):=\limsup _{k \rightarrow \infty}\left(\sup _{P(\lambda) \in \Psi_{\otimes}^{k}} \eta_{\|\cdot\| \|}[P(\lambda)]\right)^{\frac{1}{k}},
$$

where $\eta_{\|.\|}[P(\lambda)]$ is as in Definition 2.1. 
Lemma 2.12. Let $\|$.$\| be a norm on \mathbb{R}^{n}$, and $\Psi$ be a bounded set of max matrix polynomials. Then for every $k \in \mathbb{N}$,

$$
\left(\eta_{\|\cdot\|}(\Psi)\right)^{k} \leq \sup _{P(\lambda) \in \Psi_{\otimes}^{k}} \eta_{\|\cdot\|}[P(\lambda)] .
$$

Proof. Let $k \in \mathbb{N}$ be given. Since $\Psi$ is bounded, there exists a $M>0$ such that $\eta_{\|.\|}[P(\lambda)] \leq M$ for all $P(\lambda) \in \Psi$. Let $l \geq k$ be given. Write $l=k m+j$ for some $j=0,1, \ldots, k-1$. If $P_{1}(\lambda), \ldots, P_{l}(\lambda) \in \Psi$, then by Theorem 2.6(5), there exists a $M_{0}>0$ such that

$$
\begin{aligned}
\eta_{\|\cdot\|}\left[P_{1}(\lambda) \otimes \cdots \otimes P_{l}(\lambda)\right] & \leq M_{0} \prod_{i=0}^{m-1} \eta_{\|\cdot\|}\left[P_{i k+1}(\lambda) \otimes \cdots \otimes P_{i k+k}(\lambda)\right] \\
& \times \prod_{i=1}^{j} \eta_{\|\cdot\|}\left[P_{m k+i}(\lambda)\right] \\
& \leq M_{0}\left(\sup _{P(\lambda) \in \Psi_{\otimes}^{k}} \eta_{\|\cdot\|}[P(\lambda)]\right)^{m} M^{j}
\end{aligned}
$$

By setting $M^{\prime}=M M_{0}{ }^{\frac{1}{j}}$, we have

$$
\eta_{\|\cdot\|}\left[P_{1}(\lambda) \otimes \cdots \otimes P_{l}(\lambda)\right] \leq\left(\sup _{P(\lambda) \in \Psi_{\otimes}^{k}} \eta_{\|\cdot\|}[P(\lambda)]\right)^{m} M^{\prime j}
$$

Hence

$$
\begin{aligned}
\left(\sup _{P(\lambda) \in \Psi_{\otimes}^{l}} \eta_{\|\cdot\| \|}[P(\lambda)]\right)^{\frac{1}{l}} & \leq\left(\sup _{P(\lambda) \in \Psi_{\otimes}^{k}} \eta_{\|\cdot\|}[P(\lambda)]\right)^{\frac{m}{l}} M^{\prime^{\frac{j}{l}}} \\
& =\left(\sup _{P(\lambda) \in \Psi_{\otimes}^{k}} \eta_{\|\cdot\|}[P(\lambda)]\right)^{\frac{1}{k}}\left(\sup _{P(\lambda) \in \Psi_{\otimes}^{k}} \eta_{\|\cdot\|}[P(\lambda)]\right)^{\frac{-j}{k l}} M^{\prime \frac{j}{l}} .
\end{aligned}
$$

Therefore, by taking limit superior, the result holds.

Theorem 2.13. Let $\|$.$\| be a norm on \mathbb{R}^{n}$, and $\Psi$ be a bounded set of max matrix polynomials. Then

$$
\eta_{\|\cdot\|}(\Psi)=\lim _{k \rightarrow \infty}\left(\sup _{P(\lambda) \in \Psi_{\otimes}^{k}} \eta_{\|\cdot\|}[P(\lambda)]\right)^{\frac{1}{k}}
$$

Proof. Using Lemma 2.12, for all $k \geq 1$, we have

$$
\eta_{\|\cdot\|}(\Psi) \leq\left(\sup _{P(\lambda) \in \Psi_{\otimes}^{k}} \eta_{\|\cdot\|}[P(\lambda)]\right)^{\frac{1}{k}} \leq \sup _{l \geq k}\left(\sup _{P(\lambda) \in \Psi_{\otimes}^{l}} \eta_{\|\cdot\|}[P(\lambda)]\right)^{\frac{1}{l}} .
$$

So, by taking limit (as $k \longrightarrow \infty$ ) and using Definition 2.11, the result holds.

Theorem 2.14. Let $\|$.$\| be a norm on \mathbb{R}^{n}$, and $\Psi$ be a bounded set of max matrix polynomials. Then

$$
\eta_{\|\cdot\|}(\Psi)=\lim _{k \rightarrow \infty}\left(\sup _{P(\lambda) \in \Psi_{\otimes}^{k}}\|P(\lambda)\|\right)^{\frac{1}{k}}
$$


Proof. By Proposition 2.7, there exist $K_{1}>0$ and $K_{2}>0$ such that, for any $k \geq 1$,

$$
\left(K_{1} \sup _{P(\lambda) \in \Psi_{\otimes}^{k}} \eta_{\|\cdot\|}[P(\lambda)]\right)^{\frac{1}{k}} \leq\left(\sup _{P(\lambda) \in \Psi_{\otimes}^{k}}\|P(\lambda)\|\right)^{\frac{1}{k}} \leq\left(K_{2} \sup _{P(\lambda) \in \Psi_{\otimes}^{k}} \eta_{\|\cdot\| \|}[P(\lambda)]\right)^{\frac{1}{k}} .
$$

So, by taking limit (as $k \longrightarrow \infty$ ) and using Theorem 2.13, the result holds.

Let $P(\lambda)=A_{0} \oplus \lambda A_{1} \oplus \cdots \oplus \lambda^{m-1} A_{m-1}$ be a max matrix polynomial as in (1.1). We denote the set of all coefficients of $P(\lambda)$ by $\Sigma_{P}=\left\{A_{0}, A_{1}, \ldots, A_{m-1}\right\}$. For a set $\Psi$ of max matrix polynomials, consider

$$
\Psi^{\prime}=\bigcup_{P \in \Psi} \Sigma_{P}
$$

Also, we introduce the notations $S(\Psi)=\bigcup_{k=1}^{\infty} \Psi_{\otimes}^{k}$ and $S\left(\Psi^{\prime}\right)=\bigcup_{k=1}^{\infty} \Psi_{\otimes}^{\prime k}$.

Finally, we state the following two theorems.

Theorem 2.15. Let $\Psi$ be a bounded set of max matrix polynomials. Suppose that $\mu(A) \leq 1$ for all $A \in S\left(\Psi^{\prime}\right)$. Then there exists a monotone norm $\|$.$\| on \mathbb{R}^{n}$ such that $\eta_{\|.\|}[P(\lambda)] \leq 1$ for all $P(\lambda) \in \Psi$.

Proof. Since $\Psi$ is a bounded set of max matrix polynomials, by (2.6), $\Psi^{\prime}$ is also a bounded set. The inequality $\mu(A) \leq 1$ for all $A \in S\left(\Psi^{\prime}\right)$, and [15, Lemma 5], imply that $S\left(\Psi^{\prime}\right)$ is bounded. Thus by [15, Lemma 3], there exists a monotone norm $\|$.$\| on \mathbb{R}^{n}$ such that $\eta_{\|.\|}(A) \leq 1$ for all $A \in \Psi^{\prime}$. Now let $P(\lambda)=A_{0} \oplus \lambda A_{1} \oplus$ $\cdots \oplus \lambda^{m-1} A_{m-1}$ be an arbitrary max matrix polynomial in $\Psi$. Then $A_{i} \in \Psi^{\prime}$, and so $\eta_{\|.\|}\left(A_{i}\right) \leq 1$ for all $i=0, \ldots, m-1$. Hence, by Definition 2.1 , the result holds.

Theorem 2.16. Let $\Psi$ be a set of max matrix polynomials. Suppose that $\mu\left(C_{P}\right) \leq$ 1 for all $P(\lambda) \in S(\Psi)$, where $C_{P}$ is as in (2.2). Then there exists a norm $\|$. on $\mathbb{R}^{n}$ such that $\eta_{\|\cdot\|}(A)<1$ for all $A \in S\left(\Psi^{\prime}\right)$.

Proof. Let $P(\lambda) \in S(\Psi)$ be arbitrary. So there exists a $k>0$ such that

$$
P(\lambda)=P_{1}(\lambda) \otimes \cdots \otimes P_{k}(\lambda)=C_{0}^{(P)} \oplus \lambda C_{1}^{(P)} \oplus \cdots \oplus \lambda^{t} C_{t}^{(P)},
$$

where $t \in \mathbb{N} \cup\{0\}, C_{i}^{(P)}=A_{i_{1}}^{(P)} \oplus \cdots \oplus A_{i_{m}}^{(P)}$ for some $m \in \mathbb{N}$ and $A_{i_{j}}^{(P)} \in \Psi_{\otimes}^{\prime k}$. It is clear that for every $i, j$,

$$
\mu\left(A_{i_{j}}^{(P)}\right) \leq \mu\left(C_{i}^{(P)}\right)<1
$$

Since $P(\lambda) \in S(\Psi)$ is arbitrary, $\mu(A)<1$ for every $A \in S\left(\Psi^{\prime}\right)$. Hence, by [16, Theorem 2], for all $A \in S\left(\Psi^{\prime}\right), \eta_{\|\cdot\|}(A)<1$ for some norm $\|$.$\| on \mathbb{R}^{n}$.

Acknowledgement. The second author has been supported by the Mahani Mathematical Research Center of Shahid Bahonar University of Kerman, Kerman, Iran. The authors wish to express their gratitude to anonymous referees for helpful comments and useful suggestions. 


\section{REFERENCES}

1. Gh. Aghamollaei, N. Avizeh and Y. Jahanshahi, Generalized numerical ranges of matrix polynomials, Bull. Iranian Math. Soc. 39 (2013), 789-803.

2. Gh. Aghamollaei and A. Salemi, Polynomial numerical hulls of matrix polynomials, II, Linear Multilinear Algebra, 59 (2011), 291-302.

3. F.L. Baccelli, G. Cohen, G.J. Olsder and J.P. Quadrat, Synchronization and Linearity: An Algebra for Discrete Event Systems, Wiley, Chichester, New York, 1992.

4. R.B. Bapat, A max version of the Perron-Frobenius theorem, Linear Algebra Appl. 275-276 (1998), 3-18.

5. R.B. Bapat, S. Pati and S.Z. Song, Rank preservers of matrices over max algebra, Linear Multilinear Algebra, 48 (2000), 149-164.

6. R.B. Bapat and T.E.S. Raghavan, Nonnegetive matrices and Applications, Encyclopedia of Mathematics, Vol. 64, Cambridge University Press, Cambridge, 1997.

7. P. Butkovic, Max-linear Systems: Theory and Algorithms, Springer-Verlag, London, 2010.

8. R.A. Cuninghame- Green, Minimax Algebra, Lecture Notes in Economics and Mathematics Systems, Vol. 166, Springer, Berlin, 1979.

9. L. Elsner and P. Van den Driessche, On the power method in max algebra, Linear Algebra Appl. 302-303 (1999), 17-32.

10. L. Elsner and P. Van den Driessche, Max-algebra and pairwise comparison matrices, Linear Algebra Appl. 385 (2004), 47-62.

11. S. Gaubert, Methods and applications of $(\max ,+)$ linear algebra, STACS 97 (Lbeck), 261282, Lecture Notes in Comput. Sci., 1200, Springer, Berlin, 1997.

12. B.B. Gursoy and O. Mason, Spectral properties of matrix polynomials in the max algebra, Linear Algebra Appl. 435 (2011), 1626-1630.

13. B. Heidergott, G.J. Olsder and J. Van der Woude, Max plus at work: Modeling and Analysis of Synchronized Systems, A course on Max-Plus Algebra and Its Applications, Princenton University Press, 2006.

14. R. Horn and C. Johnson, Matrix Analysis, Cambridg University Press, New York, 1985.

15. Y.Y. Lur, A max version of the generalized spectral radius theorem, Linear Algebra Appl. 418 (2006), 336-346.

16. Y.Y. Lur, On the asymptotic stability of nonnegative matrices in max algrbra, Linear Algebra Appl. 407 (2005), 149-161.

17. A. Peperko, On the max version of the generalized spectral radius theorem, Linear Algebra Appl. 428 (2008), 2312-2318.

18. A. Salemi and Gh. Aghamollaei, Polynomial numerical hulls of matrix polynomials, Linear Multilinear Algebra 55 (2007), 219-228.

19. S.Z. Song and K.T. Kang, Column ranks and their preservers of matrices over max algebra, Linear Multilinear Algebra 51 (2003), 311-318.

${ }^{1}$ Department of Mathematics, Kerman Science and Research Branch, Islamic Azad University, Kerman, Iran.

E-mail address: ghasemizade.neda@yahoo.com

2 Department of Mathematics, Shahid Bahonar University of Kerman, 7616914111, KeRMAN, Iran.

E-mail address: aghamollaei@uk.ac.ir, gh_aghamollaei@yahoo.com 